Service social

\title{
Regard sur la notion de rôles sociaux. Réflexion conceptuelle sur les rôles en lien avec la problématique du processus de production du handicap
}

\section{Patrick Fougeyrollas et Kathia Roy}

Volume 45, numéro 3, 1996

Santé

URI : https://id.erudit.org/iderudit/706736ar

DOI : https://doi.org/10.7202/706736ar

Aller au sommaire du numéro

Éditeur(s)

École de service social de l'Université Laval

ISSN

1708-1734 (numérique)

Découvrir la revue

Citer cet article

Fougeyrollas, P. \& Roy, K. (1996). Regard sur la notion de rôles sociaux. Réflexion conceptuelle sur les rôles en lien avec la problématique du processus de production du handicap. Service social, 45(3), 31-54.

https://doi.org/10.7202/706736ar
Résumé de l'article

Nous présentons dans cet article les résultats d'une revue de la littérature sur les rôles sociaux, au terme de laquelle se dégagent deux grandes tendances, l'une basée sur les disciplines de la sociologie et de l'anthropologie, l'autre sur celle de la psychologie sociale. Ces tendances entrent en contradiction l'une avec l'autre, parce qu'elles partent de principes différents (approches socioculturelles vs approches individuelles) ; il s'en dégage d'une façon générale que des confusions apparaissent, notamment lorsque nous voulons situer la problématique des rôles sociaux au regard de la dimension des habitudes de vie d'une personne ayant des incapacités, et s'inscrivant dans le processus de production du handicap. Il s'agit donc de dégager les difficultés et les limites associées à l'utilisation de la notion de rôle dans le modèle conceptuel du processus de production du handicap. En étendant la problématique aux processus favorisant la participation sociale des personnes ayant des incapacités, le concept de rôle social devient alors un élément clé mais ambigu, permettant toutefois de s'interroger sur la qualité de cette participation sociale. 


\section{Regard sur la notion de rôles sociaux Réflexion conceptuelle sur les rôles en lien avec la problématique du processus de production du handicap}

Patrick FOUGEYROLLAS

Anthropologue Directeur scientifique Institut de réadaptation en déficience physique de Québec

Kathia ROY Étudiante à la maîtrise en anthropologie Assistante de recherche Institut de réadaptation en déficience physique de Québec

\section{DU CHAMP DE LA DÉVIANCE À L'ANTHROPOLOGIE DE LA SANTÉ: QUELQUES PISTES POUR UNE DÉMARCHE THÉORIQUE}

La déviance se définit habituellement à l'intérieur de paramètres où figurent les notions de légalité et de moralité et comme un écart d'une normalité définie par le social (Freidson, 1995). Dans cette perspective, la sociologie s'intéresse principalement aux phénomènes relevant de pratiques illicites diverses, telles que la délinquance et la criminalité, et où la notion de responsabilité 
individuelle est centrale. Toutefois, les travaux sur les personnes ayant des incapacités, même si ces personnes ne sont pas responsables de leur condition, ont été inclus par similarité dans ce champ général de recherche. Dans cette optique, les notions de différence, de marginalité et d'écart par rapport à la norme fonctionnelle et sociétale permettent d'insérer dans le domaine d'étude propre à la déviance la compréhension des déterminants de la participation sociale ou plus spécifiquement des rôles sociaux des personnes qui présentent ces différences.

Par ailleurs, l'anthropologie de la santé, en tentant de comprendre les modes d'intégration ou d'exclusion sociale des personnes différentes dans leur fonctionnalité, leur comportement ou leur esthétique, considère globalement les facteurs sociaux de production de la maladie et, de façon croissante depuis les années 1970, ses conséquences fonctionnelles et sociales. Dans une perspective de changement social, nous nous concentrons sur les interrelations entre les différences corporelles et fonctionnelles d'un individu et son contexte de vie dans la réalisation des rôles habituellement définis par la société. La présente analyse s'inscrit donc dans le champ de recherche portant sur $l^{\prime}$ «interaction personne-environnement ». Elle vise à clarifier le concept de rôle social comme résultat de ce processus interactif, dans un champ d'application particulier: les personnes ayant des incapacités ${ }^{1}$.

Les interventions d'adaptation et de réadaptation qui visent à développer et à maintenir les capacités fonctionnelles des personnes ayant des déficiences persistantes qui entraînent des incapacités ont amplement contribué à élargir le modèle biomédical traditionnel (Strauss, 1965 ; Myers, 1965 ; Nagi, 1965 ; Gingras, 1975). Celui-ci est centré sur la guérison par la découverte de l'étiologie de la pathologie et le traitement de l'organe ou des fonctions métaboliques pour le retour à la norme biologique. Ce modèle d'intervention est particulièrement adapté au phénomène de la maladie aiguë, mais ne l'est pas en ce qui concerne des conditions chroniques ayant un impact global sur la personne et sa vie quotidienne. L'approche globale ou holistique de la personne en adaptation-réadaptation, élaborée depuis les années 1970, a centré ses efforts autant sur le développement des capacités fonctionnelles que sur l'atténuation des conséquences psychosociales et la réalisation d'activités ordinaires correspondant au rôle social de la personne dans son milieu de vie d'origine (Bolduc, 1986; Flynn et Nitsch, 1980; Fougeyrollas, 1986 ; Joubert et Fillion, 1981 ; Wolfensberger, 1972). Toutefois, 
de manière analogue au modèle biomédical centré sur l'organe, le modèle couramment élaboré dans le système d'adaptationréadaptation demeure orienté sur la personne, qui reste essentiellement identifiée comme le centre du problème à régler, et en néglige généralement les interventions sur le contexte de vie. Ainsi, les évaluations interdisciplinaires réalisées en adaptationréadaptation n'informent pas habituellement, bien que cette situation tende à évoluer dans les années 1990, sur le degré et les déterminants physiques et sociaux de l'intégration sociale ou de la marginalisation qui sera vécue par la personne dans son milieu ordinaire de vie (Fougeyrollas, 1995).

Lorsque nous tentons de définir les déterminants sociaux de l'intégration ou de l'exclusion des individus ayant des incapacités et comment ceux-ci se traduisent dans leur réalité quotidienne, nous rencontrons très vite le concept de rôle social. D'un point de vue historique, ce concept prend plusieurs acceptions dans les milieux des sciences humaines et sociales, incitant à recenser les approches classiques qui s'y sont intéressées. Les objectifs de cette analyse sont de préciser les différentes définitions conceptuelles du rôle social ainsi que les typologies et les tendances utilisées par les chercheurs. Subséquemment, l'analyse permet de déterminer l'apport de ces diverses conceptions dans la compréhension et l'opérationnalisation du processus de production du handicap.

\section{DU PROCESSUS DE PRODUCTION DU HANDICAP À LA PROBLÉMATIQUE DES RÔLES SOCIAUX: ÉLABORATION D'UN CADRE CONCEPTUEL ET MÉTHODOLOGIQUE}

La nécessité de prendre en considération autant les variables de l'environnement que celles associées à l'individu comme déterminants de la qualité de la participation sociale des personnes ayant des incapacités a permis l'élaboration de modèles conceptuels liés à une perspective holistique. En relation avec les critiques et les efforts de révision de la Classification internationale des handicaps (CIDIH) de l'Organisation mondiale de la santé (OMS, 1988), les travaux réalisés au Québec depuis 1987, s'inspirant d'une approche interactioniste et systémique, ont permis la proposition d'un nouveau modèle: le processus de production du handicap (Fougeyrollas et al., 1996). 
Figure 1

Le processus de production du handicap : modèle explicatif des causes et conséquences des maladies, traumatismes et autres troubles

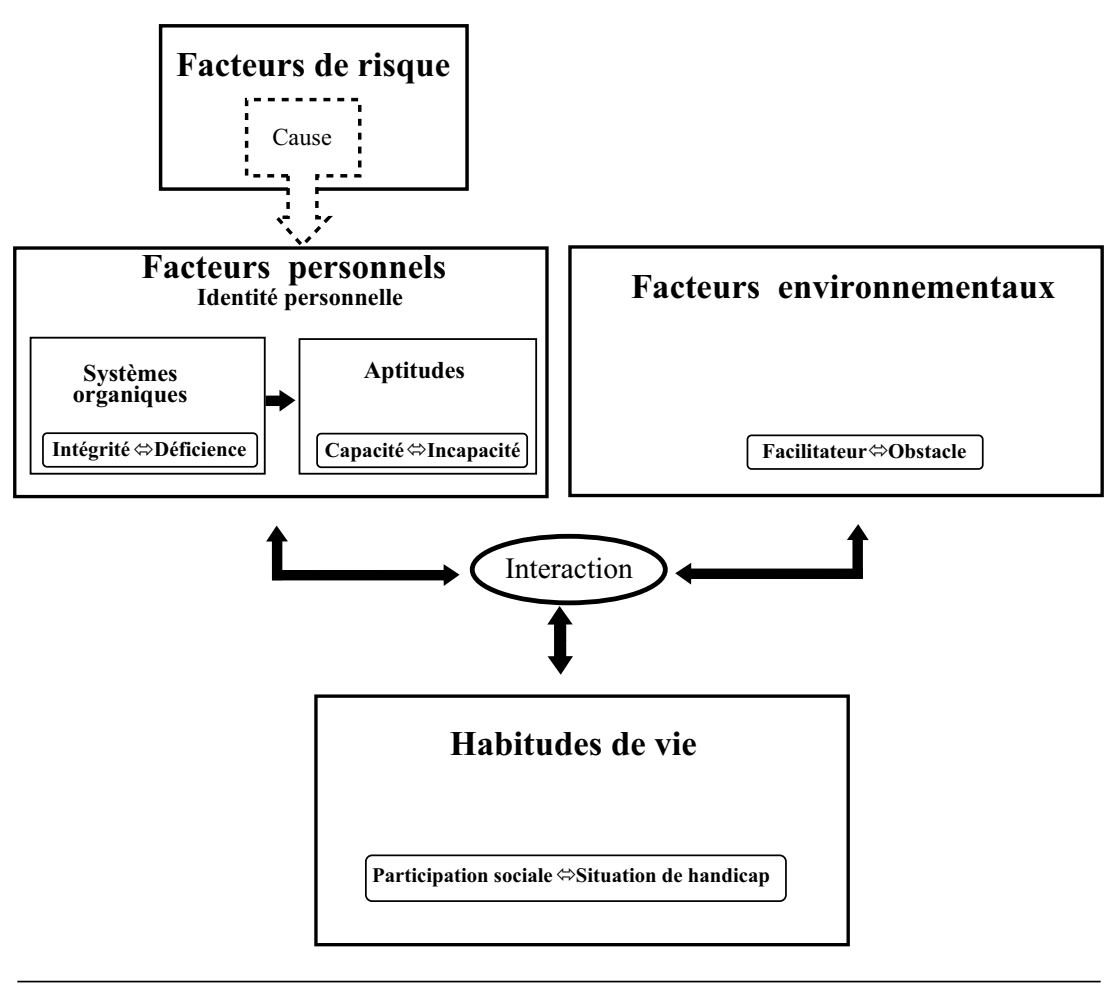

Source: Fougeyrollas, 1996.

Basé sur un modèle anthropologique ${ }^{2}$ de développement humain et sur l'interaction personne-environnement, on peut définir le processus de production du handicap comme un modèle explicatif des causes et conséquences des maladies, traumatismes et autres troubles. Comprenant un ensemble de variables individuelles et environnementales, ce processus met en lumière le mode de production d'obstacles ou de facilitateurs à la réalisation des habitudes de vie d'une personne.

Sous cet éclairage conceptuel, une situation de participation sociale ou une situation de handicap correspond au degré de réalisation des habitudes de vie, résultant de l'interaction entre, d'une part, des facteurs personnels - déficiences, incapacités et autres caractéristiques identitaires telles que l'éducation 
ou l'expérience de vie - et, d'autre part, des facteurs environnementaux constituant l'organisation et le contexte d'une société humaine sur les plans socioculturels et physiques. Selon ce modèle, le handicap ou degré de réalisation des habitudes de vie, en tant que conséquence sociale, est toujours situationnel et ne peut être compris comme une caractéristique intrinsèque de la personne. Notion clé dans notre analyse, une habitude de vie renvoie à une activité de vie quotidienne ou domestique, ou à un rôle social valorisé par le contexte socioculturel pour une personne selon son âge, son sexe, son identité sociale et personnelle. Ce sont donc les habitudes de vie qui assurent la survie et l'épanouissement d'une personne dans sa société tout au long de son existence.

Dans une perspective classificatoire, chacune des dimensions du modèle conceptuel peut donc faire l'objet d'une nomenclature. En ce qui concerne la première et la deuxième dimension des facteurs de risque et des facteurs personnels, leurs nomenclatures permettent de déterminer ce qui provoque une atteinte à l'intégrité ou au développement de l'organisme où se situe l'atteinte (sur le plan anatomique par exemple) et quelles sont les aptitudes qui sont ou non réduites. En interaction avec les facteurs personnels, la nomenclature des facteurs environnementaux, correspondant à la troisième dimension du processus, renseigne d'abord sur les dimensions socioculturelles et physiques qui déterminent le contexte d'une société, puis indique ce qui vient entraver ou faciliter la réalisation des habitudes de vie.

Figure 2

Les grandes catégories des habitudes de vie

HABITUDES DE VIE

1. Nutrition

2. Condition corporelle

3. Soins personnels

4. Communication

5. Habitation

6. Déplacements

7. Responsabilités
8. Relations interpersonnelles

9. Communauté

10. Éducation

11. Travail

12. Loisirs

13. Autres habitudes 
Constituant la quatrième dimension et le résultat du processus, les habitudes de vie, construites également sur la base d'une typologie, renseignent sur le degré de la participation sociale ou de la situation de handicap vécue par la personne. Cette explication du schéma conceptuel soulève une question: existe-t-il une typologie des rôles sociaux qui pourrait être incluse dans celle plus vaste des habitudes de vie et qui serait pertinente comme mesure validée de la participation sociale ou de la situation de handicap?

Ainsi, dans une perspective anthropologique suivant le modèle du développement humain et d'un point de vue systémique et écologique, la présente démarche vise à mieux comprendre et à catégoriser le résultat de l'interaction personneenvironnement et à le traduire en catégories d'activités construites socialement correspondant à des habitudes de vie. Pour ce faire, il faut distinguer ce qui est inhérent à l'individu (les facteurs personnels) du résultat de l'interaction personne-environnement, qui se traduit dans la réalisation de ses habitudes de vie. On comprend alors mieux la question initiale visant à vérifier si le concept de rôle social est adéquat ou suffisant pour représenter globalement ce résultat, et s'il contribue à bonifier la compréhension des conséquences sociales incluses dans le processus de production du handicap. Afin de pouvoir répondre, il est utile de définir les approches et tendances classiques et plus contemporaines qui ont abordé ce concept de rôle social et qui l'ont utilisé dans leurs analyses.

\section{LES PERSPECTIVES CLASSIQUES OU LA PRIMAUTÉ DE LA MATRICE NORMATIVE SOCIOCULTURELLE DANS LES ANALYSES SOCIOLOGIQUES ET ANTHROPOLOGIQUES}

À l'opposé des modèles biomédicaux qui situent l'individu ou même l'organe ou la pathologie comme éléments principaux des recherches, les perspectives de la sociologie et de l'anthropologie classiques font de la culture et de la société les éléments centraux de leurs études, parce qu'elles considèrent ces dernières comme des systèmes imprimant leurs valeurs et leurs normes et prescrivant des conduites relatives aux rôles joués par les individus. Le jeu de l'interaction personne-environnement, aujourd'hui largement utilisé dans les travaux de recherche, est donc peu présent dans ces perspectives classiques: c'est soit la 
matrice normative d'une société, soit l'individu qui agissent comme indicateurs. Toutefois, des perspectives classiques à celles plus contemporaines, les divers courants de la sociologie et de l'anthropologie conçoivent l'individu et la société de manière différente selon qu'ils s'intéressent au fonctionnement de l'ordre social ou à l'organisation de l'action sociale.

Ainsi, la sociologie française classique, associée aux perspectives théoriques durkheimiennes, se réclame a priori d'une conception fonctionnaliste des sociétés humaines. S'attachant à retracer la genèse de l'ordre social, l'individu apparaît comme le produit de la société dans la logique de Durkheim. Conception qualifiée de holiste, elle est cependant étroitement liée à une perspective déterministe où, comme le souligne Ogien, «[...] un système de valeurs propre à une collectivité humaine interdit à ceux qui en font partie de ne pas remplir les obligations permettant à la société qu'ils forment d'exister et de se reproduire» (Ogien, 1995, p. 104). Être patron ou employé, mère, père ou enfant signifie que l'individu remplit une fonction sociale associée à un statut, statut conféré par la société dont il est membre, cette dernière l'obligeant à respecter des normes de conduite liées à ses fonctions sociales, mettant en jeu les forces sociales et culturelles. Dans cette optique, l'individu est avant tout conditionné par sa société et il obéit généralement à des logiques qui le dépassent. En ce sens, les comportements individuels s'expliquent par les contraintes sociales qui construisent en partie les rôles sociaux que doit remplir toute personne si elle désire être partie prenante de son système social.

Alors que Durkheim met en évidence l'influence des contraintes sociales et des sanctions qui leur sont associées pour expliquer les rapports qu'entretiennent les individus avec leur société, Linton, figure centrale du courant anthropologique culturaliste étasunien (ou «culture et personnalité»), donne une place prépondérante aux processus de socialisation comme phénomènes concourant à façonner la personnalité de base et celle dite sociale. Dans cette perspective, Linton (1936) considère le rôle «[...] comme un modèle de conduite prescrit pour tous les individus détenant un même statut et accepté par eux; il exprime en fait des normes et des valeurs communes» (Gresle et al., 1990, p. 293). Lié étroitement au concept de rôle, celui de statut renvoie aux droits et aux devoirs d'un individu qui, soumis à des lois de structure imposées par le système social, évolue au sein de sa société par l'intermédiaire d'une position 
institutionnalisée. La mise en pratique de ses droits et de ses devoirs à travers certains types de conduite, consolidée par les processus de socialisation, désigne alors le rôle rempli par cet individu. En ce sens, Linton distingue le statut qui renvoie aux règles et savoir associés et le rôle, qui renvoie à l'exécution et à l'application de ces règles. À la suite de Linton, Parsons (1949) reprendra cette distinction conceptuelle entre rôle et statut et l'intégrera dans sa théorie de l'action sociale. Donnant priorité à la société qui fait figure de «noyau dur» face à l'individu, les logiques «lintonienne» et «parsonienne» donneront toutefois à la sociologie des éléments classiques d'analyse du lien qui s'établit entre l'individu et le milieu social dans lequel il s'inscrit.

Laissant peu de place à l'action et aux stratégies individuelles, les premières théories fonctionnalistes et culturalistes qui se sont intéressées à l'analyse des rôles sociaux furent cependant largement contestées en raison de leurs postulats de base jugés trop déterministes. Aussi, certains théoriciens ont critiqué le bien-fondé d'une distinction entre rôle et statut, ce qui a été notamment le cas de Nadel (1970), anthropologue britannique. Selon ce dernier, deux difficultés majeures se posent lorsque nous voulons distinguer rôle et statut. D'abord, les règles de méthode proposées par la sociologie ne précisent pas la façon d'opérer une distinction précise entre ces deux concepts. Si le fait d'être avocat relève du statut lorsque nous considérons la position tenue dans une hiérarchie sociale, il devient un rôle lorsqu'il est envisagé sous l'angle des prescriptions associées à une occupation professionnelle. Le problème réside ici en ce qu'il est difficile d'établir des distinctions entre deux aspects différents d'une même réalité renvoyant à toute action humaine déterminée en fonction de règles. La seconde difficulté réside dans le terme même de l'action humaine; on ne peut endosser un rôle chaque fois que l'on réalise une action. Par exemple, rouler à bicyclette ne fait pas automatiquement de l'individu un coureur cycliste professionnel reconnu dans son rôle. En fait, Nadel souligne que ce sont dans des activités socialement organisées que prennent naissance les rôles sociaux, ceux-ci renvoyant toujours à un engagement particulier en lien avec des attentes sociales, ce que l'action humaine ne dénote pas obligatoirement, a priori quand cette dernière prend une forme universelle (Ogien, 1995). Dans la partie ultérieure consacrée à une discussion sur les liens entre la problématique et cette revue de la littérature, nous verrons comment Nadel contribue, par son analyse des rôles et 
des statuts, à faciliter la compréhension de la segmentation que l'on fait entre rôle et activité de vie quotidienne.

Ce que soulignent ces premières analyses sociologiques et anthropologiques du rôle ainsi que leurs critiques associées, c'est que le concept de rôle relève à la fois d'éléments statiques et dynamiques. Statiques lorsque le rôle répond à une distribution de fonctions socialement admises (être parents, employeurs, étudiants, etc.); et dynamiques lorsque le rôle renferme un ensemble d'indications d'après lesquelles une personne guide son action dans une conduite acceptable aux yeux des autres (Ogien, 1995). Ainsi, c'est à partir des éléments dynamiques du rôle que l'on voit apparaître les premières analyses à tendance psychologique où sont resitués des modèles axés de plus en plus sur l'individu.

\section{DE l'ÉCOLE DE CHICAGo À LA PSYCHOLOGIE SOCIALE OU LES EFFORTS CONJUGUÉS POUR RÉINTRODUIRE L'INDIVIDU DANS LES ANALYSES}

C'est notamment en réaction à la sociologie classique de la dernière décennie du $19^{\mathrm{e}}$ siècle que se forment de nouveaux courants d'interprétations sociologique et anthropologique. Berceau de la sociologie et de la psychologie sociale étasunienne, l'École de Chicago fait d'abord référence à un ensemble de travaux de recherche élaborés entre les années 1915 et 1940, par des enseignants puis des étudiants fréquentant cette université. À partir de 1930, on utilisera fréquemment l'expression «École de Chicago " pour désigner des recherches sociologiques à caractère empirique. Qualifiée à prime abord de sociologie urbaine, l'École de Chicago ne s'intéresse pas seulement aux phénomènes migratoires et à l'intégration d'un nombre important d'immigrants à la société américaine, mais aussi à la sociologie de la famille, de la marginalité, de la déviance, etc. (Coulon, 1992). C'est à travers ces dernières perspectives que se forgent les premières théories où le rôle tient une place centrale.

Désirant rendre à l'individu une place importante dans sa relation au système social, la première génération des théoriciens de l'École de Chicago mettra en évidence l'existence d'attitudes individuelles et de valeurs partagées dans l'organisation sociale d'une collectivité. Ainsi, l'étude des attitudes relèvera généralement de la psychologie et celle des valeurs sociales, de 
la sociologie. George Herbert Mead (1934), l'un des fondateurs de l'interactionisme symbolique, institua les premiers liens entre la psychologie et la sociologie, ce qui donna lieu à la création de la psychologie sociale de l'École de Chicago.

S'inspirant à la fois d'une approche sémiotique élaborée par Charles Pierce et d'une perspective psychologique associée à Williams James, l'interactionisme symbolique puise directement à une philosophie de l'expérience. En fait, selon la perspective de Mead, "[...] la conscience des individus s'élabore à travers les interactions sociales et les processus sociaux» (Coulon, 1992, p. 13). En soulignant le caractère symbolique de la vie sociale, l'interactionisme symbolique postule que les activités interrelationnelles des individus participent à la construction de différentes significations sociales, qu'il faut alors décoder pour être en mesure de faire ensuite l'analyse de la macro-société. En ce sens, Mead fut l'un des premiers à faire la synthèse entre l'approche macrosociologique de la sociologie classique et celle associée à la microsociologie où l'individu est central, grâce entre autres à la notion de «soi » qu'il considérait comme «[...] l'intériorisation du processus social par lequel des groupes d'individus interagissent avec d'autres» (Coulon, 1992, p. 14).

Accordant une place théorique à l'acteur social en tant qu'interprète du monde qui l'entoure, la pensée de Mead peut se définir, selon Bergeron (1974), par une sorte de "parallélisme» entre les rôles joués et la conscience de jouer ces rôles. Cette conscience, pour Mead, conduit à un processus de socialisation qui incorpore le langage et les interactions des individus à travers ce langage. C'est Mead qui, le premier, introduit le terme de «role-taking» comme concept central de son approche. Ainsi, le role-taking de Mead se définit par «[...] un processus mental qui a pour but d'anticiper les réactions d'autrui dans l'interaction "lui-moi" »(Bergeron, 1974, p. 98-99). En fait, le «moi social» de James deviendra un préalable à l'émergence du roletaking de Mead, donnant à penser qu'il est une forme complexe de la connaissance de soi, une sorte d'interaction déplacée et intériorisée dans l'acte de penser. Ce concept est aussi vu comme le prolongement du processus de socialisation et des acquisitions des significations sociales. Pour Mead, le rôle se définit d'une manière dramaturgique et ne concerne que «l'attitude mentale» dans «son moment psychologique» ou «pré-comportemental». Enfin, une autre notion clé dans la perspective de Mead est ce concept «d'autrui généralisé». Pour l'auteur, «[...] l'individu ne reflète qu'une perspective ou un aspect du modèle d'ensemble» 
(Bergeron, 1974, p. 108), ce modèle d'ensemble faisant bien sûr référence à la société et plus globalement à la culture dans laquelle il s'insère.

À l'inverse des premières analyses sociologiques, les perspectives de Mead s'inspirent en partie d'une approche psychologique classique du rôle amené par William James autour des années 1890. Étant le "pôle dur» de la société, l'individu est considéré comme un être rationnel faisant des choix stratégiques, ces derniers influençant par la suite l'organisation du système social. Pour James, l'être humain est multi-orienté; il joue donc plusieurs rôles, selon les situations, les gens rencontrés, etc. Ainsi, de nouveaux concepts sont créés: "moi social» et «type social», entre autres, qui désignent un ordre de réalité où «[...] le psychisme individuel rencontre les processus sociaux» (Bergeron, 1974, p. 8). Ce sont, parmi d'autres, ces termes qui sont à la base de la recherche et de la création de la première définition psychologique du rôle. Apport considérable, plusieurs théoriciens sociologues reprendront à leur compte la définition de James du «moi social» qui propose que «[...] l'individu est un ensemble de plusieurs personnes » (Bergeron, 1974, p. 9). Repris puis abandonné par Linton (1936), le «moi social» de James est en fait une «image projetée dans l'opinion des autres » et peut être associé au principe de reconnaissance (recognition). Associant les notions de statut et de rôle, il fait référence à une «[...] sorte de statut conféré à un individu dans son milieu, après qu'il se fut conformé à certaines exigences» (Bergeron, 1974, p. 80).

Demandant invariablement l'approbation d'autrui, cette notion centrale dans les analyses de James définit et renferme déjà en partie la notion de statut selon la perspective de Park et Burgess (1931), deuxième génération de sociologues de l'École de Chicago fortement influencés par l'interactionisme symbolique de Mead. Pour ces derniers, le concept de statut s'exprime par une «[...] sorte de gratification sociale accordée à un individu en échange de sa conformité aux exigences du groupe, et sans laquelle il ne possède aucune identité sociale» (Bergeron, 1974, p. 9). Pour ces théoriciens, l'individu assimile le rôle à une fonction et ils définissent la personnalité comme «la source et l'organisation des traits» qui déterminent le rôle de l'individu dans le groupe auquel il appartient. À partir de cette définition, Park et Burgess se pencheront sur l'étude des conflits de rôles, notamment en ce qui concerne la marginalité, les phénomènes de racisme, etc. (Rocheblave-Spenlé, 1969). 


\section{LE RÔLE COMME FONCTION SOCIALE ET CONDUITE INDIVIDUELLE OU EN TANT QUE RÉSULTAT DE L'INTERACTION PERSONNE-ENVIRONNEMENT}

Inspirés à la fois par la sociologie et la psychologie sociale, Park s'intéressant à la sociologie urbaine et Burgess à la sociologie de la famille, ils en viendront à dire que «l'organisme urbain et familial» comprend des individus qui ont des rôles fonctionnels mais aussi dramaturgiques dans une logique où les rôles sont associés à des phénomènes sociaux spécifiques. Influencée sans doute par Burgess et à mi-chemin entre la sociologie et la psychologie sociale puisque inspirée par un courant béhavioriste, Katharine Lumkin (1933) s'intéresse à trois aspects du rôle: le rôle dramatique, la fonction sociale du rôle et les conduites individuelles reliées aux divers statuts des individus partageant un même système social. Pour Lumkin, les rôles servent au maintien du groupe (par exemple les fonctions liées au travail). Plus spécifiquement en lien avec le milieu familial, d'où l'influence de Burgess, les études et recherches de Lumkin définissent le rôle comme un «[...] terme général pour décrire certains devoirs, prérogatives et attitudes que les personnes ayant une même fonction dans un groupe tendent à avoir en commun» (Rocheblave-Spenlé, 1969, p. 17). Inspirée aussi de la sociologie et de l'anthropologie, Lumkin avance l'idée que les rôles sociaux dépendent des coutumes, des normes et valeurs de la société globale, tout en définissant les codes et normes relatifs à une certaine position dans cette même culture ou société.

Antérieurement, Baldwin (1895), autre théoricien important, influençait particulièrement la psychologie sociale de l'École de Chicago et la psychologie française, en posant les fondements analytiques que Lumkin allait reprendre. À certains égards, il rejoint Park dans sa conception de «jouer un rôle» et James pour qui le rôle social n'est pas une fonction, mais une apparence, une façade, une interaction avec autrui (Bergeron, 1974). S'intéressant principalement au développement de l'enfant, Baldwin mentionne que les besoins de base se situent sur le plan psychologique. Par la suite, l'enfant devient peu à peu un être social. Les mécanismes de cette double connaissance (de soi et des autres) et les réactions aux autres comme à lui-même constituent essentiellement le premier pas vers les rôles sociaux que l'enfant aura à remplir tout au long de sa vie (Rocheblave-Spenlé, 1969). Il devient ainsi adulte, c'est-à-dire 
le représentant d'un type social, en jouant un ou des rôles particuliers.

\section{QUELQUES INFLUENCES DES TENDANCES CLASSIQUES SUR LES RECHERCHES RÉCENTES}

De Durkheim à George Herbert Mead, en passant par Linton, Park ou Burgess, les réflexions actuelles sur la place des rôles dans l'analyse de faits sociaux sont largement influencées par ces auteurs classiques. Ainsi, à partir de travaux consacrés se sont construites des analyses et des typologies qu'utilisent encore aujourd'hui plusieurs chercheurs, autant dans les disciplines de la sociologie et de l'anthropologie que de la psychologie sociale.

Selon Chapuis et Thomas (1995), il est possible d'établir une distinction initiale entre les divers types de rôles, et ce, en fonction de certains critères. Ces auteurs avancent l'hypothèse que certains rôles seraient inéluctables parce qu'ils sont liés à des attributs biologiques tels que le sexe et l'âge. Parce que l'individu doit accepter cet état de fait, on parle alors de rôles assignés ou imposés. En ce sens, les rôles sont plus ou moins choisis par la personne, ce qui constitue un premier critère de classification. Ensuite, un rôle rassemble un certain nombre de comportements prescrits; l'individu a toutefois la possibilité d'adapter ce rôle et de le jouer d'une certaine manière, selon la marge de liberté qu'il se fixe par rapport au rôle joué. Deuxième critère de classification des rôles, on distingue les rôles prescrits des rôles réels. Suivant cette logique de classification, nous retrouvons donc quatre types de rôles, à savoir: 1. Le rôle imposé par les attributs de l'individu, tels que l'âge et le sexe; 2 . Le rôle acquis, qui est fonction des actes de l'individu, tel son rôle professionnel; 3 . Le rôle prescrit, qui correspond à l'ensemble des comportements et conduites attendus selon le statut de l'individu; 4 . Le rôle réel, qui renvoie au rôle prescrit mais mis en acte de façon subjective. En fait, cette logique de classification implique trois ordres de relations entre les différents aspects du rôle qui découlent d'abord des attentes liées au rôle, puis de la conception personnelle de ce rôle et, enfin, de l'interprétation de ce dernier (Chapuis et Thomas, 1995). Cette manière de classifier les différents types de rôle a été inspirée par Erving Goffman (1961), qui s'intéressa plus particulièrement à la question du rapport entre 
rôle et identité et qui, à la suite de George Herbert Mead, insista sur le caractère interactionnel du processus d'acquisition des rôles.

Ainsi, selon Goffman, le rôle renfermerait trois dimensions, la première étant normative, la seconde, typique et la troisième, interprétative. Dans la foulée des travaux de Goffman, Ogien (1995) souligne que la dimension normative se définit «[...] par les règles de conduites idéales qu'un individu devrait respecter pour assurer la fonction qu'un rôle particulier est censé remplir». La dimension typique «[...] se rapporte aux attributs et qualités qui sont couramment associés à la personne qui remplit tel ou tel rôle sans qu'ils n'entrent dans sa définition officielle». Ogien (1995) donne l'exemple d'un policier qui, afin de jouer adéquatement son rôle professionnel, devrait posséder une qualité telle que le «sang-froid». Enfin, la dimension de l'interprétation $d u$ rôle renvoie à «[...] l'interaction au cours de laquelle un individu tient la place qui lui échoit, en s'évertuant à ne déroger ni aux normes idéales, ni à celles relevant des conceptions typiques»(Goffman, 1961, dans Ogien, 1995, p. 106).

Dans un autre ordre d'idées, on ne peut passer sous silence un ouvrage important qui s'est entièrement consacré à l'analyse des rôles sociaux, d'un point de vue toutefois plus idéologique que théorique. Dans les années 1980, Wolfensberger et Thomas (1983) font des recherches, entre autres, sur le thème de l'intégration sociale des personnes ayant des déficiences mentales ou physiques. Sous ce thème de recherche, ils se sont intéressés non seulement aux rôles sociaux, mais aussi à la valorisation de ces derniers, liés à différents services humains. L'ouvrage Passing. Programme d'analyse des systèmes de services. Application des buts de la valorisation des rôles sociaux (Wolfensberger et Thomas, 1983) est devenu l'outil de référence par excellence dans les milieux des services sociaux. Créé afin d'améliorer l'image que l'on a des personnes «différentes", cet ouvrage a effectivement largement contribué à une meilleure compréhension des situations que vivent les personnes ayant des incapacités et, de ce fait, a collaboré à leur intégration sociale. D'après Wolfensberger et Thomas, le rôle social renvoie à un

[...] modèle de comportement socialement attendu et habituellement défini afin de faciliter la réalisation de(s) fonction(s) particulière(s) qui sont généralement attribuées à ou assumées par un individu et qui reflètent partiellement le statut social de cette personne. Les rôles 
sociaux offrent des attentes, des tâches, des responsabilités et des privilèges à la personne qui les remplit. Par exemple, on s'attend à ce que le rôle social de «mari » soit tenu par un homme adulte, que les hommes adultes ayant ce rôle soient responsables d'aider à fonder et à approvisionner une famille et qu'ils puissent profiter des privilèges d'une relation maritale et parentale. Ordinairement, les personnes dont le rôle social est le plus élevé et qui sont les plus valorisées socialement auront davantage de choix et de rôles sociaux. Par exemple, les personnes valorisées peuvent habituellement choisir le travail qu'ils désirent faire, s'ils désirent se marier, quels vêtements ils vont porter, etc. D'un autre côté, les personnes ayant un statut social inférieur ou qui sont socialement dévalorisées se font souvent imposer un ou des rôles sociaux (p. 32).

On constate que cette définition du rôle social comprend à la fois une tendance sociologique et une tendance psychologique, plaçant les auteurs dans une démarche «hybride», ce qui correspond, on le verra plus loin, à notre conception, qui doit inclure à la fois les éléments psychologiques et sociaux dans la compréhension des processus sous-tendant leur accomplissement. En effet, Wolfensberger et Thomas définissent le rôle en lien avec ce qui est "socialement attendu» (approche de la psychologie sociale), mais aussi en lien avec des fonctions spécifiques qui refléteront le statut de l'individu (approche sociologique).

À partir de cette définition et de l'analyse sociale en découlant, les théoriciens ont alors élaboré une classification des rôles sociaux, classification leur permettant de jeter les bases des principes de la normalisation (et, dans une version plus récente, la valorisation des rôles sociaux, dont il est ici question). Malgré l'apport considérable de ces recherches, les principes de la normalisation suggèrent qu'il existe une idéologie sous-jacente attestant l'existence de quelque chose d'habituel, d'une réalité générale qui sous-tend des normes perçues comme des vérités objectives, et où sont inclus des rôles sociaux prescrits par la société. Cette logique, associée au modèle normatif dominant dans nos sociétés, se conçoit mal sans faire le lien avec la persistance d'une normalité qui est jugée partagée par tous les membres d'un même système social. Mais comme on sait que la déviance est construite entre autres par l'institutionnalisation, rendre les gens «normaux», faire croire qu'une apparence 
«ordinaire» peut contribuer à intégrer normalement une personne relève d'une nouvelle forme de violence, puisque les différences sont niées, évacuées au profit d'une standardisation limitative. Par contre, il faut redire que le postulat initial de Wolfensberger et Thomas (1983) est adéquat dans une société où les différences sont écartées: si la personne ayant des incapacités désire participer au projet collectif, la façon «idéale» de le faire passe probablement par ses rôles sociaux, notamment par le rôle de travailleur, associé largement aujourd'hui dans notre société aux processus d'intégration sociale. Cependant, le rôle de travailleur pour une personne ayant des incapacités se fera généralement dans un contexte adapté à ses besoins particuliers, d'où l'importance de replacer non seulement l'influence de l'environnement sur l'individu, mais aussi les ascendants de ce dernier sur l'environnement sociétal.

Cette revue de la littérature entourant le concept de rôle social reste incomplète dans la mesure où il a fallu faire un «découpage» et un choix parmi les auteurs abordés. Par exemple, on aurait pu examiner plus longuement les liens entre les rôles sociaux et l'identité qu'introduisait Erving Goffman dans les années 1960, ou encore traiter plus particulièrement des processus interactionnels et ce, dans une perspective qui tienne compte de la complexité et du dynamisme des phénomènes situationnels. Toutefois, les quelques théories mises en relief donnent l'occasion de mettre en évidence un certain nombre d'éléments de discussion en lien avec les rôles sociaux et le processus de production du handicap.

\section{QUELQUES ÉLÉMENTS DE DISCUSSION}

D'entrée de jeu, il est intéressant de constater l'évolution conceptuelle de la notion de rôle social. Des tendances classiques à celles plus contemporaines, les théoriciens ont d'abord abordé cette notion d'un point de vue déterministe où le système social était central. Puis l'individu a ensuite été progressivement replacé dans les analyses, jusqu'à être à son tour au cœur des théories, notamment dans les travaux de la psychologie sociale. Aujourd'hui, grâce notamment aux approches interactionistes et écologiques, le jeu des deux composantes - système social et individu - est généralement replacé dans une interaction «environnement-individu», rééquilibrant en quelque sorte les «deux 
principaux pôles» des analyses théoriques. Parallèlement à cet état de fait, une tendance comparable se profilait dans le domaine de la recherche en santé. D'abord centrée principalement sur des approches biomédicales où l'individu était conçu avant tout comme un ensemble d' "organes» ou comme une "machine», on voit de plus en plus s'esquisser des travaux où la personne est devenue un être beaucoup plus complexe, influencée par sa culture et l'influençant à son tour, tout en évoluant dans des contextes de significations précis. En tant qu'outil d'analyse, les modèles interactifs permettent maintenant de faire un «compromis» entre l'individu et l'environnement physique et culturel dans lequel il se meut, en plus de favoriser des analyses beaucoup plus dynamiques.

Dans le modèle conceptuel du processus de production du handicap (voir fig. 1), l'individu est placé dans la catégorie des facteurs personnels. En mettant en relation les facteurs de risque avec non seulement les déficiences ou les incapacités, mais aussi avec l'individu "comme un tout», correspondant en fait au développement de la personne, il est possible d'y placer l'ensemble des variables personnelles, telles que l'identité, le vécu, etc., qui sont le fruit de l'histoire de ce développement et dans lequel il existe une rupture spécifique due à un événement pathologique ou encore à des conditions héréditaires, congénitales ou de développement. On considère dès lors qu'il existe des éléments intrinsèques du développement de la personne, qu'il existe des éléments extrinsèques de l'individu, se retrouvant dans l'environnement; on estime de plus que l'interaction entre ces éléments provoque un résultat, traduisible entre autres par les rôles sociaux, ces derniers étant englobés - comme on l'a mentionné antérieurement - dans la catégorie des habitudes de vie où, en tant qu'agir, ils participent à la fois à la construction des facteurs personnels et des facteurs environnementaux. Cependant, phénomène souvent négligé, l'ensemble de ce processus doit être replacé dans le temps, parce que tout est en constante évolution, en changement. Le seul moyen de mettre en valeur l'influence des variables personnelles et environnementales dans les mécanismes situationnels est de les situer dans un processus dynamique, où l'interaction est considérée elle aussi comme mouvante et complexe, selon les espaces, le temps et les sociocultures envisagés.

Ce processus systémique isole en quelque sorte le «cumul» du développement de la personne, c'est-à-dire l'ensemble des 
facteurs personnels se traduisant par un résultat qui vient construire l'identité. D'un point de vue dynamique, l'ensemble des rôles sociaux que joue l'individu déterminent la construction de ce résultat, comme si le «cumul» des rôles sociaux fabriquait l'identité personnelle, elle aussi résultant de l'interaction personne-environnement. Pour bien saisir ces éléments, on peut se référer au processus du développement biologique, qui rejoint d'une certaine manière la conception de Baldwin. Ce processus débute avant même la naissance. Ensuite, l'enfant développe ses propres traits de personnalité et est socialisé petit à petit à travers son environnement (familial, social, physique, culturel). Ce qu'il fait lorsqu'il est enfant le construit pour l'avenir, le prépare à vivre sa vie d'adulte et à jouer toute une série de rôles sociaux. Cependant, l'enfant évolue dans un environnement socioculturel spécifique. Dès lors, il n'y a pas que les facteurs personnels qui auront une influence sur la construction de son identité. Comme le mentionnait Lumkin (1933), les rôles sociaux dépendent des coutumes, des normes et valeurs d'une société globale. C'est ce qu'on appelle la "matrice normative» d'un système social. On se rend compte alors que les rôles sociaux ne sont pas, dans leur totalité, une caractéristique intrinsèque de l'individu, mais un résultat entre les facteurs personnels et les facteurs environnementaux qui viendront fabriquer l'expérience de vie de la personne. En ce sens, les rôles sociaux sont à la frontière floue entre l'influence de l'environnement (milieu de vie, normes, valeurs, etc.) et l'expérience de l'individu (rôle en tant que conduite, manière d'interpréter et de construire sa réalité, ses activités de vie quotidienne, etc.).

À un deuxième niveau d'explication lié cette fois directement au cadre conceptuel, à la lumière des analyses et réflexions sur le concept de rôle social, on arrive à la conclusion qu'il faut prendre en considération les deux grandes perspectives théoriques qui se sont intéressées à la problématique du rôle, soit celle de la sociologie et de l'anthropologie et celle de la psychologie sociale. Elles sont en effet pertinentes toutes deux, selon la fluctuation des événements dans une réalité donnée. Toutefois, comme le rôle social peut figurer comme un résultat ou une conséquence sociale, la perspective fonctionnelle couvre probablement mieux l'interprétation que l'on fait des phénomènes sociaux comprise dans les rôles, dans la mesure où l'individu y est resitué en tant qu'acteur adoptant certaines stratégies qui faciliteront son insertion dans le système social. À cet égard, il 
ne s'agit plus d'adopter une perspective uniquement fonctionnelle, mais de la replacer dans une dynamique situationnelle, où les processus interactionnels font alors figure d'éléments centraux. C'est seulement à ce titre que l'on peut rendre compte de manière adéquate de la complexité des phénomènes sociaux et plus particulièrement des éléments mis en jeu dans les activités associées aux rôles sociaux.

Par contre, lorsqu'il s'agit d'aborder la problématique du rôle d'une manière plus spécifique, quelques difficultés se posent. Ainsi, les différentes définitions du concept de rôle restent insuffisantes pour expliquer tout le vaste champ des habitudes de vie, ainsi qu'il est proposé dans le modèle conceptuel du processus de production du handicap, notamment en ce qui concerne les activités de vie quotidiennes.

En fait, selon la logique du processus de production des situations de handicap et notamment ce qui concerne les habitudes de vie, une activité de vie quotidienne ne comprend pas obligatoirement une dimension interpersonnelle. Or, le concept de rôle selon la psychologie sociale repose essentiellement sur cette dimension. Ainsi, pour qu'il y ait rôle, il doit y avoir cette interrelation entre deux ou plusieurs personnes. Par exemple, l'activité de vie quotidienne que suppose «manger» ne peut être comprise comme un rôle social; pourtant, cette activité relève de la dimension culturelle puisqu'elle suppose des rituels (de propreté, de politesse, etc.) ainsi qu'une certaine convention sociale (avec qui je mange, seul ou en groupe, dans un lieu public ou chez moi, etc.). Cet exemple illustre donc les difficultés que l'on a à saisir l'ensemble d'une réalité sociale à partir du seul concept de rôle, puisqu'il existe des activités de vie quotidienne qui s'inscrivent dans la dimension culturelle sans toutefois aborder le champ des rôles sociaux. Cette dernière analyse rejoint en quelque sorte les critiques que faisait Nadel, particulièrement sur les distinctions entre rôle et action.

Autrement dit, lorsqu'on tente d'insérer à l'intérieur du schéma conceptuel du processus de production du handicap le rôle social comme concept global pour expliquer le résultat de l'interaction personne-environnement, on fait rapidement face à des difficultés relevant de la segmentation entre rôle social et activité de vie quotidienne. En fait, selon la définition qui varie suivant le domaine de la réadaptation, une activité de vie quotidienne, c'est-à-dire une activité de base comme se vêtir, manger, se laver, etc., ne s'insère pas nécessairement dans des 
processus d'interrelations, ce que dénote obligatoirement le rôle social. Par exemple, malgré le fait que se vêtir soit une activité quotidienne attendue socialement, puisqu'elle procède de règles, elle ne s'inscrit pas à un premier plan dans une interaction entre des individus. Ainsi, l'action qui consiste à se vêtir n'est pas un rôle social, mais est bel et bien une activité de vie quotidienne. Partant de ce principe, c'est à bon droit que l'on n'élabore pas une nomenclature ou une classification des rôles sociaux, puisque ces derniers, englobant trop peu d'activités de vie quotidienne, limitent nos analyses au lieu de les enrichir. En ce sens, le rôle n'explique pas complètement le lien entre les activités de vie quotidienne et la dimension sociale dans lequel s'inscrit le processus de production des situations de handicap. Sa définition reste insuffisante, car elle ne recouvre pas la totalité du champ social qu'abordent les habitudes de vie.

\section{CONCLUSION}

S'inspirant d'une perspective globale de l'être humain, le cadre théorique et méthodologique privilégié dans cette réflexion est basé à la fois sur une approche écologique, interactioniste et systémique. Accentuant la nécessité de considérer autant les variables de l'environnement que celles associées à l'individu comme déterminantes de la qualité de la participation sociale des personnes ayant des incapacités, le schéma conceptuel du processus de production du handicap met en lumière le mode de production d'obstacles ou de facilitateurs à la réalisation des habitudes de vie d'une personne. Partant de ces premiers éléments, on a tenté de mieux comprendre et catégoriser le résultat de l'interaction personne-environnement et de le traduire en catégories d'activités construites socialement, correspondant à des habitudes de vie. Subséquemment, on s'est interrogé, d'abord, sur la pertinence du concept de rôle social comme éventuel indicateur validé de ce résultat, puis à savoir s'il contribue ou non à l'éclaircissement de l'influence des variables sociales incluses dans le processus de production du handicap. En dernière instance, on s'est demandé s'il peut ou non faire l'objet d'une typologie validée qui pourrait être comprise dans celle plus vaste des habitudes de vie et qui deviendrait pertinente dans l'appréciation de la qualité de la participation sociale ou de la situation de handicap. 
Afin de répondre aux questions soulevées par cette problématique de recherche, une revue de la littérature non exhaustive autour du concept de rôle social a permis un retour sur les auteurs qui l'ont abordé et qui l'ont utilisé dans leurs analyses, de même que sur leurs théories. D'une façon générale, il s'en dégage deux grandes tendances: l'une est basée sur la sociologie et l'anthropologie et l'autre sur la psychologie sociale, la première privilégiant une approche où prime le système social et la seconde, l'individu. D'abord conçu d'un point de vue déterministe où le système social était central, l'individu a ensuite été progressivement replacé dans les analyses, jusqu'à être à son tour au cœur des théories, notamment dans les travaux de la psychologie sociale. Dans un exercice de «rééquilibrage» des "deux principaux pôles» des analyses théoriques, éléments de l'environnement et éléments individuels sont aujourd'hui replacés dans une interaction personne-environnement. Le rôle social y apparaît déterminé à la fois par les normes et valeurs d'une société et par l'expérience individuelle, c'est-à-dire par les choix de vie, la manière d'interpréter et de construire sa réalité et ses activités de vie quotidiennes. En somme, les rôles sociaux apparaissent à la frontière des facteurs personnels et environnementaux, se traduisant dès lors dans la réalisation des habitudes de vie.

Concept flou qui a relevé de différentes définitions selon les perspectives théoriques des chercheurs du domaine social et humain, le terme de rôle social reste ambigu et partiel. Partiel puisqu'il ne rend pas compte systématiquement, dans la logique du modèle conceptuel du processus de production du handicap, de l'ensemble des activités de vie quotidienne puisque ces dernières ne supposent pas obligatoirement une interrelation entre les individus. Partiel, de ce fait, puisqu'il ne peut remplacer entièrement les habitudes de vie. Partiel, enfin, puisqu'il n'arrive pas à rendre compte globalement de ce résultat de l'interaction entre l'environnement et l'individu dans la réalisation des habitudes de vie. 


\section{Notes}

1. Le terme incapacité est ici utilisé dans le sens de toute personne ayant des restrictions dans ses activités physiques ou mentales de façon significative et persistante à la suite d'une maladie ou d'un traumatisme ou, encore, en raison de conditions héréditaires, congénitales ou de développement.

2. Lorsque nous utilisons le terme "anthropologique», nous faisons référence essentiellement aux modèles systémique et écologique, qui voient dans la construction de l'être humain le caractère inséparable de l'interaction entre la personne et son environnement physique et social.

\section{Références bibliographiques}

BADley, E., et A. TEUNANT (1995). «Disability, Resources, Role Demands and Mobility Handicap», Disability and Rehabilitation, vol. 17, $\mathrm{n}^{\circ} 3-4$, p. 159-168.

BADLEY, E. (1995). "The Genesis of handicap : models of disablement, and role of external factors ", Disabilities and Rehabilitation, vol. $17, \mathrm{n}^{\circ} 2$, p. 53-61.

BALDWIn, J. (1895, 1968). Mental Development in the Child and the Race,

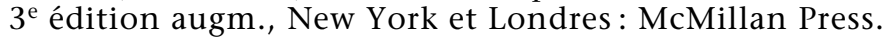

BERgeron, A. (1974). Les origines de la notion de rôle en sociologie et en psychologie sociale, Thèse. Sainte-Foy: Québec, Université Laval.

Bolduc, M. (1986). Analyse de la cohérence de politiques québécoises à l'égard des personnes ayant des incapacités, Québec: ministère de la Santé et des Services sociaux, Direction de l'évaluation des programmes.

Bonte, P., et M. IZARD (dir.) (1992). Dictionnaire de l'Ethnologie et de l'Anthropologie, Paris: Presses universitaires de France.

Borgatta, E., et M.L. BorgatTA (1992). Encyclopaedia of Sociology, vol. 3, Seattle: University of Washington Press.

CHAPUis, R., et R. ThOMAS (1995). Rôle et statut, Paris : Presses universitaires de France. (Coll. Que sais-je?)

Coulon, A. (1990). L'ethno-méthodologie, Paris: Presses universitaires de France. (Coll. Que sais-je ?)

Coulon, A. (1992). L'École de Chicago, Paris: Presses universitaires de France. (Coll. Que sais-je?)

Flynn, R.J. et K.E. Nitsch (1980). Normalization, Social Integration and Community Services, Baltimore: University Park Press.

Fougeyrollas, P., R. Cloutier, H. Bergeron, J. Côté, M. Côté et G. ST-MiCHEL (1996). Révision de la proposition québécoise de classification: Processus de production du handicap, Québec: CQCIDIH.

Fougeyrollas, P. (1986). "Processus de production du handicap et lutte pour l'autonomie des personnes handicapées »(Dossier), Anthropologie et sociétés, vol. 10, $\mathrm{n}^{\circ} 2$. 
Fougeyrollas, P. (1995). Le processus de production culturelle du handicap. Contexte sociohistorique du développement des connaissances dans le champ des différences corporelles et fonctionnelles, Québec: Éd. Patrick Fougeyrollas.

FreIDSON, E. (1995). «Disability as Social Deviance» dans Sociology and Rehabilitation, Washington, DC: American Sociological Association.

GINGraS, G. (1975). Combat pour la survie, Paris : Éd. Robert Laffont.

GOFFMAN, E. (1961). Encounters : two studies in the sociology of interaction, Indianapolis : Bobbs-Merrill.

Gresle, F., M. PANOFf, M. PERrin et P. Trippier (1990). Dictionnaire des Sciences Humaines: Sociologie, Psychologie sociale, Anthropologie, France: Nathan.

JAmes, W. (1950). The Principles of Psychology (1890), New York: Dover Publ.

JARY, D., et J. JARY (1991). The Harper Collins Dictionary of Sociology, Harper Collins Publishers.

JOUBERT, P., et R. FILLION (1981). «L'influence des conditions ambiantes sur la construction du handicap: pratiques québécoises en réadaptation", Santé mentale au Canada, vol. 29, n 4.

LinTon, R. (1936). The Study of Man, New York, Appleton Century (trad. fr.: De l'homme, Paris : Ed. de Minuit, 1968).

LUMKIN, K. (1933). The Family, a Study of Member Roles, Chapel Hill: University of North Carolina Press.

Mauss, M. (1966). Sociologie et Anthropologie, Paris: Presses universitaires de France.

MeAD, G.H. (1934). Mind, Self and Society (trad. fr. : L'esprit, le soi et la société, Paris: Presses universitaires de France, 1963).

MinAIRE, P. (1983). «Le handicap en porte-à-faux», Prospective et Santé, $\mathrm{n}^{\circ} 26$, p. 39-46.

MYERS, J.K. (1965). «Consequences and Prognoses of Disability», Sociology and Rehabilitation, American Sociology Association, Marvin B. Sussman, Cleveland: Western Reserve University, n ${ }^{\circ} 11, \mathrm{p} .33-51$.

NADEL, S. (1957, 1970). La Théorie de la structure sociale, Paris : Éd. de Minuit.

NAGI, S.A. (1965). «Some conceptual issues in disability and rehabilitation», Sociology and Rehabilitation, Ohio: Ohio State University Press, p. 5-6.

OgIEn, A. (1995). Sociologie de la déviance, Paris : Éd. Armand Colin.

ORGANISATION MONDIALE DE LA SANTÉ (OMS) (1988). Classification internationale des handicaps : déficiences, incapacités et désavantages. Un manuel de classification des maladies, Paris : INSERM-CTNHERHI.

PAPALIA, D. et S.W. OLDS (1988). Introduction à la psychologie, McGrawHill.

PARK, R.E. et E.W. BURGESS $(1931,1969)$. Introduction to the Science of Sociology, Chicago: University of Chicago Press.

PARSONS, T. (1937, 1949). The Structure of Social Action, New York: The Free Press. 
Paulhan, F. (1992). Les Caractères, Paris: Éd. Félix Alcan.

PERLMAN, H.H. (1973). La personne: l'évolution de l'adulte et de ses rôles dans la vie, Paris : Éd. du Centurion.

RoCHEBlAVE-SPENlÉ, A.M. (1969). La notion de rôle en psychologie sociale: étude historico-critique, $2^{\mathrm{e}}$ éd. augm., Paris : Presses universitaires de France.

Soder, M. (1988). "The Concept of Handicap. A Comparison between ICIDH Definitions and a Swedish Definition of Impairment, Disability and Handicap» (Rapport), Conseil suédois de planification et de coordination de la recherche, vol. 88, $\mathrm{n}^{\circ} 1$.

STRAuSS, R. (1965). "Social Change and the Rehabilitation Concept», Sociology and Rehabilitation, American Sociology Association, Marvin B. Sussman, Cleveland: Western Reserve University, $\mathrm{n}^{\circ}$ 1, p. 1-33.

TARDE, G. (1979). Les lois de l'imitation. Étude sociologique (1843-1904), Paris : Éd. Félix Alcan.

Willems, E. (1970). Dictionnaire de sociologie, $2^{\mathrm{e}}$ éd. augm., Paris : Éd. M. Rivière et Cie.

Wolfensberger, W., et S. ThOmas (1983). Passing. Programme d'analyse des systèmes de services. Application des buts de la valorisation des rôles sociaux, $2^{\mathrm{e}}$ éd. augm., Canada: Les Communications Opell.

Wolfensberger, W. (1972). The Principle of Normalization in Human Services, Toronto: National Institute of Mental Retardation. 